\title{
Televisión de libre recepción y televisión por cable:
}

\section{perspectivas de desarrollo*}

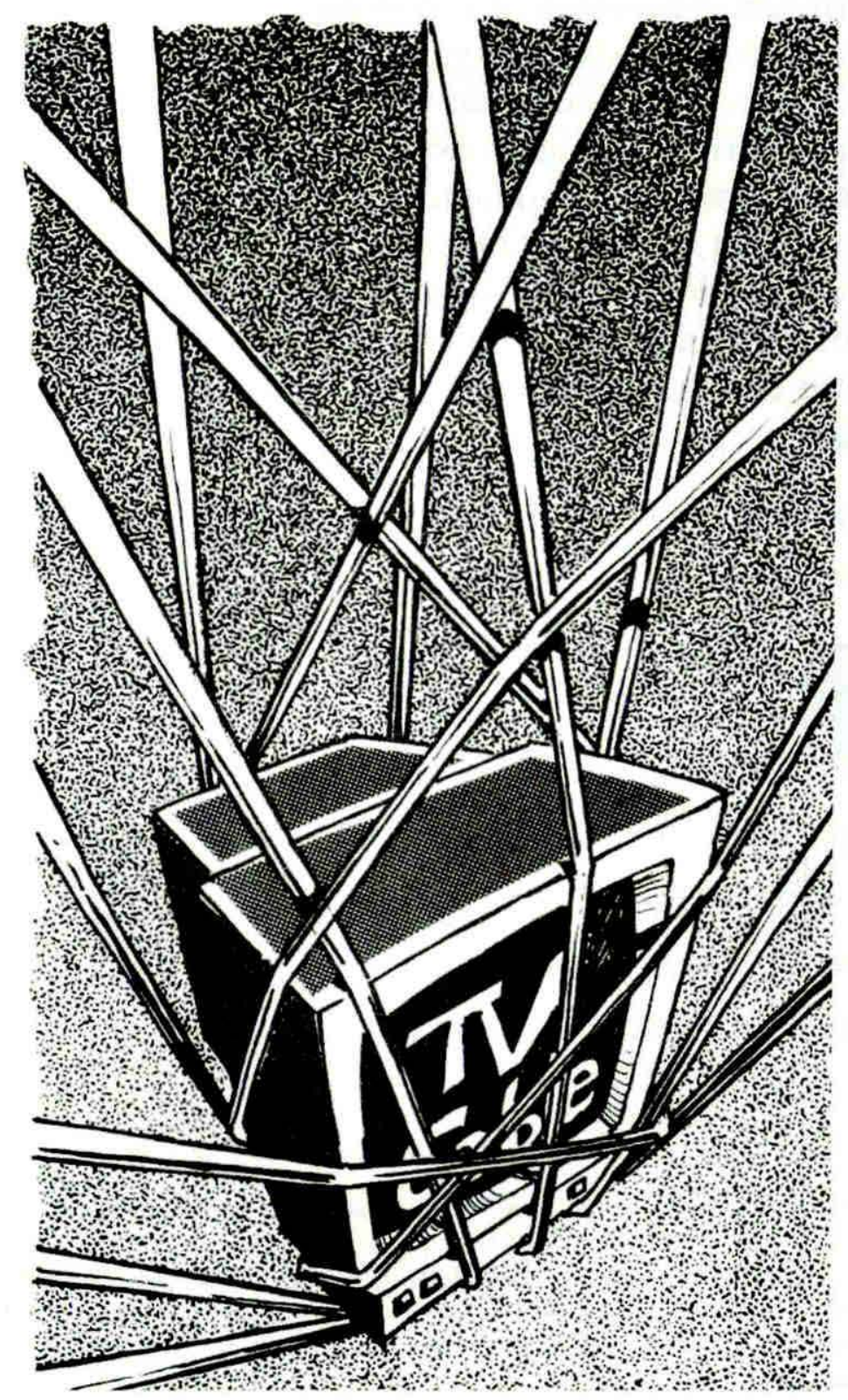

La incorporación de la televisión por cable es sólo el más reciente de los muchos cambios que ha experimentado el sistema televisivo chileno en los últimos años. Hacia el futuro, las perspectivas de desarrollo son enormes $e$ interesantes, y traerán muchas novedades, tal vez más profundas y sorprendentes que las que hemos ya conocido.
Por Jorge Navarrete Martínez

\section{UNA PERSPECTIVA HISTORICA}

A

ntes de entrar de lleno al tema de este artículo, creo conveniente reseñar algunas constantes en el desarrollo de los medios de comunicación social (MCS), que nos ayuden a poner el momento que vivimos, tan preñado de variadas posibilidades futuras, en una perspectiva histórica de más largo plazo.

\section{Las hipótesis catastróficas}

Ha sido una constante histórica en el desarrollo de los MCS que, cada vez que la investigación científica- y tras ella la innovación tecnológica- han introducido 
un nuevo MCS o alterado significativamente las condiciones de uno o más de los ya existentes, se han levantado voces que han sostenido lo que, en algunas entrevistas de prensa recientes, he llamado las "hipótesis catastróficas"; hoy en día, nuevas voces comienzan a alzarse para plantear estas mismas tesis.

Entiendo por "hipótesis catastrófica" aquella que, ante el desarrollo de un nuevo MCS o de un cambio importante en la tecnología de los existentes, siempre ha pronosticado la desaparición, o a lo menos la reducción a la insignificancia, de las tecnologías o MCS hasta entonces existentes.

Así, en distintas épocas se ha pronosticado, siempre con una seguridad seudo-científica, la desaparición o futura irrelevancia de los libros, de los diarios y revistas, del cine y de la radiodifusión.

Hoy el turno le toca a la TVLR, y la nueva hipótesis catastrófica señala que el desarrollo futuro de la de la CATV y, en especial, el de la "super carretera electrónica" y de la televisión interactiva, harán -si no desaparecer- reducir drásticamente la importancia de la industria de la TVLR.

Yo estoy convencido de que esto no sucederá, lo que no significa que la TVLR no deberá enfrentar cambios importantes en el futuro inmediato, como veremos más adelante; pero la experiencia histórica indica que esos cambios no tendrán un signo tan crítico.

\section{Lo efectivamente sucedido}

La historia enseña que, ante el desarrollo científico-tecnológico en general, y el de los MCS en particular, las industrias afectadas nunca tienden a desaparecer.

Más aún, rara vez sufren una reducción de su tamaño absoluto, aunque sí pueda verse afectada, a veces muy fuertemente, su posición relativa frente a otras industrias.

No disponemos del tiempo necesario para demostrar exhaustivamente esta constante histórica, lo que me obligaría a adentrarme en la historia de las industrias editorial, de la prensa escrita, del cine, de la radiodifusión y de la televisión.

Pero permítanme recordarles, a lo menos a aquellos de ustedes que tengan la edad suficiente para recordar los inicios de la televisión en Chile, que en la década de los sesenta se afirmó taxativamente, por muchos, que el desarrollo y expansión masiva de este nuevo medio reduciría a la insignificancia, si es que no condenaría a la desaparición, a la industria de la radiodifusión.

Ello no sucedió: la radio siguió existiendo. No sólo eso: la radiodifusión avanzó tecnológicamente, creó nuevas formas de servicios y, contra todas las prediccio- nes catastróficas, hoy existen mucha más empresas de radiodifusión en Chile que las que había hace treinta años, y la industria en su conjunto ha aumentado en términos absolutos su contribución al Producto Nacional Bruto (PNB), aunque haya disminuido su participación relativa en él y en el del sector de los medios de comunicación.

El ejemplo de la radiodifusión no ha sido un caso aislado, sino, antes bien, un caso más de una constante histórica ante situaciones semejantes.

\section{Enseñanzas que se derivan de la historia}

¿Porqué se ha dado esta constante histórica?. En particular, ¿porqué no desapareció sino que continuó desarrollándose la radio, ante la aparición en la escena social y económica de un medio que, como la televisión, parecía ofrecer lo esencial de la radio, el sonido, pero agregando un nuevo y poderosísimo elemento, la imagen?.

La respuesta consiste en que, ante la aparición de una nueva tecnología y/o de un nuevos MCS, ni las tecnologías existentes dejan de seguir desarrollándose, ni los antiguos MCS- y, con ellos, sus pro-

Cada vez que la investigación científica y la innovación tecnológica han introducido un nuevo MCS o alterado significativamente las condiciones de uno o más de los ya existentes, se han levantado voces que han sostenido "hipótesis catastróficas"; hoy en día, nuevas voces comienzan a alzarse para plantear estas mismas tesis. 
La televisión de libre recepción no desaparecerá, aunque deberá enfrentar cambios importantes en el futuro inmediato; la experiencia histórica, en todo caso, indica que esos cambios no tendrán un signo tan crítico.

pietarios, ejecutivos y trabajadores- se sientan a esperar el resultado de la "crónica de una muerte anunciada".

Por el contrario, la nueva competencia se transforma en desafío y, sin pretender ignorar el impacto de lo nuevo, las industrias ya existentes se reorientan y se concentran en desarrollar sus ventajas comparativas, abandonando o reduciendo su actividad en aquellas áreas donde se concentran las ventajas competitivas del nuevo MCS o de la nueva tecnología.

Así sucedió, para volver al mismo ejemplo, con la industria de la radiodifusión, que desarrolló nuevas tecnologías, tanto de transmisión como de recepción; creó nuevas modalidades, como la de frecuencia modulada y el sonido estereofónico; reorientó sus servicios hacia las áreas en que tenía ventajas competitivas, como la música, la información y los servicios a sus auditores, y, por último, abandonó o redujo significativamente áreas donde tenía ventajas comparativas la televisión, como los programas en vivo desde estudios con público, la transmisión de novelas y otros programas seriados, etc.

\section{PERSPECTIVAS DE DESARROLLO DE LA IELEVISION DE LA LIBRE RECEPCION}

A la luz de estas constantes históricas y frente al actual desarrollo y próxima expansión masiva de la CATV, así como a las perspectivas futuras de ella de proveer nuevos y valiosos servicios a su público, me atrevo a asegurar que la TVLR ni desaparecerá ni se reducirá en términos absolutos, aunque sí sufrirá importantes cambios y, probablemente, perderá importancia relativa en relación a las industrias afines.

Para saber hacia adonde irán esos cambios, y a la luz de la experiencia histórica, quizás las mejor forma sea preguntarse dónde están las ventajas comparativas de la TVLR y, también, dónde están las de la CATV.

\section{Ventajas competitivas relativas de la TVLR y de la CATV}

Las ventajas comparativas relativas de la TVLR pueden encontrarse, me parece, en tres terrenos: primero, en lo referente a su público; segundo, en lo referente a sus contenidos programáticos $\mathrm{y}$, tercero y muy dependiente de las anteriores, en lo referente a su fuente de financiamiento.

\section{Ventajas competitivas relativas en lo referente a público}

Las ventajas competitivas de la TVLR en lo referente a público son de dos tipos, uno cuantitativo y otro cualitativo.

Calidad potencial de telespectadores: Aunque uno de los cambios significativos en la CATV ha sido el comprender, con cierto atraso, que se trata de un servicio masivo y no elitista, es un hecho -en Chile y en el mundo- que la TVLR tendrá siempre una cantidad potencial de telespectadores mayor que la CATV. Al respecto, es conveniente tener presente los siguientes hechos:

- La gran cobertura geográfica de las principales redes de TVLR:

-En el caso de las más extensas de ellas, Televisión Nacional de Chile (TVN), ella supera el $98 \%$ de la población nacional, porcentaje altísimo incluso en comparación con paises desarrollados.

-En el caso de la segunda más extensa, Universidad Católica de Chile, Corporación de Televisión (TV-UC), ya supera el 90\%, mientras la tercera Megavisión, ya se acerca o supera el $80 \%$.

- La población chilena se distribuye aproximadamente de la siguiente manera, según lugar de residencia: un 70,6\% vive en ciudades de más de 100.000 habitantes: un $11,6 \%$ vive en ciudades de menor número de habitantes $\mathrm{y}$, por último, un $17,8 \%$ vive en áreas rurales. 
- Con una hipótesis de cableado (home-passed) potencial de 60 y 40 y $0 \%$, en las tres categorías definidas más arriba, se alcanzaría un cableado total de hogares donde vive un $47 \%$ de la población; si, sobre esa base, se supone una penetración efectiva de $60 \%$, la CATV lograría una cobertura potencial de hogares donde vive el $28,2 \%$ de la población.

- Aún aumentando las hipótesis de cableado a 75,50 y $0 \%$ respectivamente, y la depenetración a un $75 \%$, la CATV lograría una cobertura potencial de hogares donde vive el $44,1 \%$ de la población.

Puede concluirse que la cobertura potencial de la TVLR será de dos a tres veces mayor que la de CATV. Naturalmente, debe subrayarse el adjetivo potencial ya que, primero, las cifras anteriores no son de audiencias reales (medidas ya sean en share o rating) y, segundo, por ser cifras para industria en su conjunto y no de un canal determinado.

Como veremos después, lo primero es importante, ya que hay base empírica para señalar que los telespectadores que disponen de CATV tienen un comportamiento de audiencia significativamente distinto de los que sólo tienen TVLR.

Tipo de audiencia: En esta materia, existen significativas diferencias entre la TVLR y la CATV, derivadas de a naturaleza del medio, de su forma de financiamiento y de los hábitos de los telespectadores.

Pidiendo excusas por no detenerme a citar las bases empíricas correspondien- tes, entre otras razones porque algunos de los hechos son de sentido común, las principales diferencias pueden resumirse en los siguientes hechos:

- Aunque la composición de audiencia por estratos socio-económicos varía entre los distintos canales, la TVLR tiende a reflejar la estructura socio-económica de la población (salvo la sub-representación del estrato E), mientras la audiencia de la CATV tiende, por el contrario, a sobre-representar los estratos $\mathrm{ABC} 1, \mathrm{C} 2$ y $\mathrm{C} 3$;

- Los telespectadores de TVLR ven menos horas de televisión, tanto en días de semana como en sábados y domingos, que los que disponen de CATV;

- La TVLR generalmente busca, y muchas veces obtiene, audiencias masivas y no segmentadas o, en todo caso, audiencias semi-masivas parcialmente segmentadas; por el contrario, la CATV busca audiencias no necesariamente masivas pero siempre fuertemente segmentadas. Dicha en otras palabras, "la TVLR busca una mayoría y la CATV colecciona $m u$ chas minorías".

\section{Las ventajas competitivas relati-} vas en lo referente a contenidos

Las ventajas competitivas relativas a la TVLR en lo referente a contenidos de programación son más subjetivas, pero parece haber cierto grado de acuerdo en que ellas se concentran en la programación de origen nacional y, dentro de ella, especialmente en los programas informativos.

\section{La programación nacional en general}

Al menos mientras la industria de la CATV no dé en Chile el paso de ofrecer, como en otros paises, diferentes servicios para grupos específicos de clientes, la TVLR chilena seguirá teniendo ventajas comparativas sobre la CATV en materias de programación nacional, desde los menos dos puntos de vista:

La historia enseña que, ante el desarrollo científico-tecnológico en general, y el de los medios en particular, las industrias afectadas nunca tienden a desaparecer. Más aún, rara vez sufren una reducción de su tamaño absoluto, aunque sí pueda verse afectada, a veces muy fuertemente, su posición relativa frente a otras industrias. 
La televisión de libre recepción generalmente busca, y muchas veces obtiene, audiencias masivas y no segmentadas o, en todo caso, audiencias semi-masivas parcialmente segmentadas; por el contrario, la televisión por cable busca audiencias no necesariamente masivas pero siempre fuertemente segmentadas.

- En cuanto a la cantidad, la programación de origen nacional continuará representando una proporción mucha más alta de su oferta total (sobre $50 \%$ en los dos canales más importantes, que la de la CATV (5\% en el mejor de los casos), y

- Con la sola, pero importante, excepción del fútbol profesional, la calidad de la producción nacional de la TVLR es hoy día, y seguirá siendo por bastante tiempo, muy superior a la de la CATV, situación en que no solo influye la notoria diferencia de experiencia y de recursos técnicos y humanos calificados, sino también en las bases económicas distintas de las dos industrias.

\section{La programación informativa en particular}

Lo señalado recién sobre la producción nacional en general se aplica, en forma especial, a la programación informativa.

- Desde luego, por el momento la CATV chilena no ofrece nada comparable a los noticieros de la TVLR, varios al día, y con un fuerte énfasis en el acontecer nacional; aunque objeto de una creciente crítica a los noticieros de TVLR por su tendencia progresiva a privilegiar el magazine por sobre las verdaderas noticias, los noticieros de los principales canales de TVLR chilenos siguen gozando de prestigio y audiencia, y aún no enfrentan competencia de la CATV.

- Quizás tan importante como la ventaja comparativa señalada, y más difícil aún de revertir por la CATV, es la que representan los otros programas informativos de la TVLR, en especial en en género de los reportajes especiales en profundidad, reportajes a la geografía física y humana de Chile y otros similares.

\section{Ventajas competitivas en lo referente a \\ financiamiento publicitario}

Las ventajas competitivas relativas de la TVLR en lo referente a financiamiento publicitario son las más complejas de predecir en su evolución futura, pero parece que, por un tiempo al menos, serán las siguientes:

\section{Cantidad de publicidad}

Aunque en los principales canales de TVLR chilenos existe una evidente "sobresaturación publicitaria", cuyos efectos negativos perciben no sólo los telespectadores sino, crecientemente, también los avisadores, es evidente que la CATV tendrá siempre mayores limitaciones en esta materia, lo que es natural por demás dado su carácter de televisión de pago, a lo que se agregan las restricciones a la publicidad en varias señales satelitales.

\section{Tipo de público-objetivo}

Se trata aquí de una ventaja comparativa mucho más discutible pero, sobre todo, susceptible de erosionarse en el futuro. En efecto:

- La TVLR es, y seguirá siendo por un buen tiempo, un mejor soporte publicitario para productos de consumo masivo, de decisión de compra no segmentada y de precios absolutos medios-bajos y bajos.

- Por otra parte, la CATV es ya, y lo será cada vez más, un mejor soporte publicitario para productos de consumo selectivo, de decisión de compra en tipos de clientes específicos y de precios absolutos medios-altos y Altos.

\section{La inercia de agencias de publici- dad y avisadores}

En el corto plazo, será la ventaja más importante en materia publicitaria de la TVLR sobre la CATV, pero también la más frágil; pero hoy es un hecho que:

- Las agencias de publicidad tienden a tener un comportamiento muy conservador en sus recomendaciones de medios a los clientes, y así ha sido siempre ante la aparición de nuevas alternativas, comportamiento que, en algunos casos, se mantiene incluso frente a la evidencia 
empírica de lo que es más conveniente para sus clientes.

- Esto está comenzando a cambiar. y la evolución parece ser mucho más rápida en los avisadores, en especial en los medianos y grandes, entre los cuales ha habido una notoria profesionalización del marketing, empujada por una generación de nuevos ejecutivos generales y de productos.

\section{Factores institucionales de la industria de la CAIV}

Por último en materias de publicidad, la TVLR se seguirá beneficiando por un tiempo de algunos factores institucionales de la CATV en esta materia, en especial la falta de métodos sistemáticos, continuos e independientes de medición de audiencia de CATV, a nivel de empresas y de señales, y el aún escaso profesionalismo de las áreas comerciales de las empresas de CATV, que tienen un corto período de existencia.

\section{Hipótesis probables sobre el desarrollo futuro de la TVLR}

Sobre la base de las diferencias entre la TVLR y la CATV, en especial de las ventajas competitivas relativas de cada una, me corresponde entrar a formular hipótesis sobre el desarrollo futuro de la VTLR en Chile.

Reiterando que se trata de hipótesis de trabajo y no de conclusiones definitivas, correré el riesgo de aventurar a conti- nuación las tendencias que preveo en la TVLR en Chile, en particular en materia de: número de canales que existirán; tipos de canales que progresarán, subsistirán o decaerán; cambios en los contenidos programáticos y cambios en la propiedad de los medios.

\section{Número de canales de IVLR}

En esta materia, mi hipótesis fundamental es que el número de canales de TVLR en Chile no aumentará, tendiendo más bien a disminuir, dependiendo de las transformaciones que algunos de ellos se decidan o no a acometer, de lo que se tratará más adelante.

Esta hipótesis de deriva, en lo fundamental, de los siguientes hechos:

Tamaño y evolución de la inversión publicitaria en Chile: Aunque la inversión publicitaria tiene elasticidad positiva en relación al PNB y, por lo tanto, de mantenerse las tasas de crecimiento del país de los últimos años, la inversión publicitaria total crecerá como porcentaje del PNB, debe considerarse que el tamaño absoluto de la inversión publicitaria no es función ni del número de canales de TVLR ni de su oferta publicitaria.

Con la sola excepción del fútbol profesional, la calidad de la producción nacional de la televisión de libre recepción es hoy día, y seguirá siendo por bastante tiempo, muy superior a la de la televisión por cable, situación en que no sólo influye la notoria diferencia de experiencia y de recursos técnicos y humanos calificados, sino también en las bases económicas distintas de las dos industrias. 
Por el momento la televisión por cable chilena no ofrece nada comparable a los noticieros de la televisión de libre recepción, con varias ediciones al día y con un fuerte énfasis en el acontecer nacional.

pos de canales de TVLR, los que creo serán de tres tipos:

\section{Canales nacionales de programa- ción general y no segmentada}

Ser esto ha sido la aspiración, hasta ahora, de todos los canales de TVLR en Chile, pero la experiencia de más de treinta años es consistente en señalar, por los factores que se apuntaron más arriba, que tendrán a agudizarse en el futuro, que es probable que subsistan y prosperen dos -o, a lo más tres, dependiendo de sus posiciones relativas- de este tipo de canales.

Un seguro primer candidato a permanecer en este selecto grupo es, naturalmente, TV-UC, que ha liderado casi ininterrumpidamente el mercado por más de tres décadas; aunque esta posición se haya debido, en ciertos períodos, a la ausencia de verdaderos competidores, es un hecho que TV-UC tiene la continuidad ejecutiva, el prestigio institucional, la lealtad de una parte importante de la audiencia, la mejor dotación de recursos materiales y financieros y un buen número de recursos humanos calificados, factores todos que debieran asegurar su futuro. Su factor de riesgo puede estar en la tendencia a la inmovilidad y en la falta de propensión al riesgo que, generalmente, se derivan de un liderazgo prolongado.

Un segundo candidato es TVN que, superada la crisis integral que vivió en la década de los ' 80 y dotada de una nueva institucionalidad que concitó un amplio acuerdo en todos los sectores, se ha ido consolidando cada día más como empresa y como medio de comunicación, habiendo recuperado la credibilidad y el pluralismo conque fue originalmente creado a fines de los '60. Su mayor fuerza es su excelente personal profesional, que ha demostrado ser un factor a veces más que compensatorio de su menor dotación de recursos materiales. Su factor de riesgo puede estar en la tendencia, al parecer muy difícil de resistir, del poder político a poner en riesgo su independencia.

De haber lugar para un tercer canal nacional de programación general no segmentada, no cabe duda que quien parece haberse ganado ese lugar es Megavisión, el primero de los canales de TVLR privados del país. Después de un comienzo no demasiado prometedor, en especial comparado con las expectativas que sus propietarios habían creado en avisadores y agencias de publicidad, el canal tuvo un cambio importante en materia del público al cual orienta preferentemente sus pro- gramas, el que coincidió con la venta de un $49 \%$ de sus acciones a Televisa, participación que hace pocos días se ha anunciado, se reducirá a no más del $10 \%$. Su factor de riesgo puede estar en la injerencia demasiado directa de sus dueños, lo que parece no haber creado las bases para la conformación de un cuerpo ejecutivo profesional y estable.

Parece que será entre estas tres entidades que se jugará la "división de honor" de la TVLR chilena. Esta competencia puede adquirir un carácter dramático si la inversión publicitaria -sea por su monto, sea por su distribución- no fuera suficiente para financiar los crecientes costos de operación de tres canales de este tipo; en tal caso, el destino del que quede tercero puede ser crítico.

Aún si esto ocurre, y que me perdonen mis muchos y buenos amigos de los otros canales de TVLR, creo altamente improbable que uno de los restantes cuatro (Chilevisión, La Red, UCV-TV y Telenorte) pueda llegar a entrar en esta liga. Se requeriría demasiado talento, demasiado tiempo y demasiado dinero, y ninguna de estas tres cosas sobra.

\section{Canales de programación segmen- tada}

Sin embargo existe un futuro, y quizás un buen futuro, para esos restantes canales de TVLR ya existentes y aún para otros que se creen en el futuro.

Mi tercera hipótesis de trabajo es que veremos, en el futuro inmediato, el desarrollo de un segundo tipo de canales de TVLR, caracterizados por orientarse a seg- 
mentos específicos de público, por trabajar con muchos menores costos de explotación y por aprovechar bien su alta audiencia en uno o más "públicos-objetivos" claramente segmentados para obtener una inversión publicitaria que, sin ser demasiado importante como proporción del total, les permita sobrevivir e, incluso, ser buenos negocios.

No se trata, por cierto, de una idea nueva, sino uno que ha sido implementada, muchas veces con éxito notable, en la TVLR de otros paises. En Chile, este fenómeno se ha dado ya en otros medios de comunicación social, donde cada vez es mayor -y más exitosa- la presencia de diarios especializados, que han venido a sumarse a las mucho más antiguas revistas especializadas. Como anticipábamos en el análisis histórico, esta estrategia fue la fundamental en la sobrevivencia y progreso de la radiodifusión, con la creación de las radios FM y la creciente segmentación de las AM. En cierto modo, esto ya ha comenzado a pasar el la TVLR en Chile, sea por designio, sea por necesidad.

Hay dos canales que, desde siempre, estuvieron marcados por una segmentación regional (UCV-TV y Telenorte), aunque han vivido en un marco de gran estrechez que, en el caso del segundo, ha sido más bien de crisis permanente.

Hay otros dos canales (Chilevisión y La Red) en que después de una larga agonía como canal de la Universidad de Chile el primero, y de sucesivos cambios de propiedad y administración el segundo, han quedado definitivamente bajo el control de capital extranjero. Es pronto aún para saber lo que ello implicará para cada uno, pero creo que su futuro no está en intentar, un vez más, "alcanzar a los grandes".

Pero la experiencia más interesante de este nuevo tipo de canal que se proyecta es el Canal 2, Rock \& Pop, que, ocupando la última frecuencia VHF disponible en Santiago, ha orientado desde el comienzo a su proyecto en esta línea de programación segmentada, en su caso, dirigiéndose al público adolescente y adulto joven. Esta clara definición, unida al éxito que sus propietarios y ejecutivos han tenido en radios AM y FM líderes en sus especialidades, nos hace esperar con expectación este nuevo experimento televisivo.

\section{Canales regionales}

Un tipo especial de segmentación lo constituyen los canales regionales. A la azarosa vida de los dos ya existentes, ya me he referido más arriba.

Sin embargo, existe una segunda alternativa de TVLR regional, la derivada de la segmentación, a ciertas horas del día, de los canales nacionales de programación general no segmentada en redes regionales. Pese a que la experiencia más antigua de las que sobreviven es el canal de TV-UC en Concepción, no cabe duda que la experiencia más sistemática, valiosa y exitosa en este campo es la de TVN. Esta empresa pública creó en 1990 una gerencia de regiones que, con el apoyo sistemático del directorio y la dirección ejecutiva, ya ha organizado, puesto en funcionamiento y financiado ocho centros regionales, con sede en Copiapó, La Serena, Valparaíso, Rancagua, Talca, Concepción, Temuco y Punta Arenas, que transmiten diariamente noticieros propios, han comenzado a producir otros programas regionales y, aunque distante ene el tiempo, no es utópico pensar que algún día TVN se transforme en la primera red nacional de centros regionales de televisión.

\section{Reorientación de los contenidos programáticos de la IVLR}

Aunque en los principales canales de televisión de libre recepción chilenos existe una evidente sobresaturación publicitaria, es evidente que la CATV tendrá siempre mayores limitaciones en esta materia, lo que es natural por demás dado su carácter de televisión de pago, a lo que se agregan las restricciones a la publicidad en varias señales satelitales. 
En el futuro inmediato veremos el desarrollo de un segundo tipo de canales de televisión de libre recepción, caracterizados por orientarse a segmentos específicos de público, por trabajar con mucho menores costos de explotación y por aprovechar bien su alta audiencia en uno o más públicos-objetivos claramente segmentados.

Además de los probables cambios en el número y tipo de canales, creo que en la TVLR se producirá una importante reorientación de sus contenido programáticos, con la acentuación de ciertas tendencias que ya se vienen expresando desde hace un tiempo y motivada, una vez más, por la necesidad de aprovechar sus propias ventajas comparativas $\mathrm{y}$, la vez, reconocer las ventajas comparativas de la CATV.

Me atrevo a aventurar, a este respecto, las siguientes hipótesis de trabajo:

Aumento de la importancia de la producción nacional: Es la primera y obvia respuesta de la TVLR al desafío creciente de la CATV y en menor medida, al frente crecimiento del video hogareño.

Tendrá que pasar un tiempo, y llevarse adelante cambios significativos en la CATV, antes que esta industria pueda ofrecer una programación nacional significativa, en cantidad y calidad.

La TVLR tiene aquí una de sus grandes ventajas, a través de programas en que el público chileno se reconoce y se siente interpretado, lo que se demuestra cada día con el lugar de privilegio de los mejores programas nacionales en los ratings de la TVLR.

Pérdida de importancia de la programación extranjera envasada: Es el cambio correlativo natural de la tendencia anterior y viene expresándose desde hace ya tiempo. La "edad de oro" de las series norteamericanas terminó, ya hace años. Más recientemente, es notoria la pérdida relativa de importancia del cine en la TVLR. Esta tendencia se incrementará, ya que la TVLR le es imposible competir -en cantidad, variedad y oportunidad- con las señales de cine de los servicios básicos ya existentes en la CATV, para no hablar de lo que llegará en materia de señales de pago mensual adicional (canales premium) y de programas de pago adicional, en sus variantes de oferta fija (pay per view) y de oferta abierta (pay on demand). Es posible que se exceptúen de esta tendencia las telenovelas extranjeras que, aunque de mucho menor éxito que las nacionales, seguirán formando parte de la programación complementaria de la TVLR.

Aumento de la importancia de la programación periodística: Dentro del aumento general de la importancia de la programación nacional de la TVLR, Creo que es también una hipótesis razonable la de suponer una especial importancia futura al género más obviamente "nacional" posible, la programación informativa. Aquí los elementos centrales serán programas como:

- Los noticieros, en sus distintas ediciones diarias.

- Los programas periodísticos especializados, que ojalá no sean sólo los deportivos.

- Los reportajes especiales en profundidad, al estilo de "Informe Especial" de TVN o "Contacto" de TV-UC en temáticas generales, o "La Tierra en que Vivimos" y "Al Sur del Mundo" de los mismos canales, en temáticas especiales.

- Otros géneros de programas "de realidad", especialmente los de dramatización de hechos reales.

Tendencia a la propiedad única, muchas veces con fuerte presen-

cia extranjera, de múltiples medios de distinto tipo

En un orden distinto de materias, quiero entregar una última hipótesis sobre el desarrollo de la TVLR que, en realidad es más bien de los MCS en general: me refiero a la tendencia a la concentración en las mismas manos -generalmente pocas, muchas veces con fuerte presencia extranjera- de múltiples medios de comunicación de distinto tipo, tendencia que se ha dado en llamar por el anglicismo "multimedia"

La concentración de la propiedad de los MCS no es nueva, ni en el extranjero no en el extranjero ni en Chile, donde 
siempre ha sido particularmente notoria en la prensa escrita. A veces este hecho ya existente, se han sumado dos tendencias nuevas. La primera, es a que la concentración de propiedad rebase los ámbitos de MCS específico para abarca varios: diarios, revista, radios AM y FM, canales de TVLR, empresas de CATV, editoriales, etc. La segunda, es que este proceso de concentración de la propiedad va acompañado por una creciente participación de capital extranjero, fenómeno que ha sido especialmente rápido y masivo en el caso de la televisión, tanto en TVLR como en CATV.

Cuando se discuten estos temas -lo que no ocurre ni muchas veces, ni públicamente, como con otros temas importantes en el Chile de hoy- se tiende a decir que se trata de una tendencia mundial que es inútil tratar de contrarrestar. No es éste el momento no la tribuna para manifestar mi desacuerdo con esta posición resignada, cuando no entusiasta. Sólo quiero señalar que estas tendencias plantean interrogantes muy de fondo que tienen que ver, entre otros, con temas tan vitales como la posibilidad real de una política democrática, la existencia del pluralismo de todo orden y la presentación de nuestra identidad nacional básica.

\section{Conclusiones sobre el desarrollo futuro de la TVLR}

En resumen, mis hipótesis fundamentales sobre el desarrollo futuro de la TVLR son las siguientes:
- Ni los cambios tecnológicos, ni la expansión de la CATV ni, siquiera, el desarrollo de la "super carretera electrónica" y la televisión interactiva, harán desaparecer a la TVLR, la que es posible incluso- que crezca en términos absolutos, aunque pierda posiciones relativas.

- Para que ello sea posible, la TVLR tendrá, como otros MCS en el pasado, que transformarse para aprovechar sus propias ventajas comparativas y no desconocer las ventajas comparativas de los otros MCS, en especial la CATV.

- Esas ventajas comparativas se refieren, materia de público, a que cuantitativamente la TVLR seguirá teniendo exclusividad en ciertas áreas no adecuadas para cable de las ciudades grandes y medianas, en los centros urbanos pequeños y en las áreas rurales, y cualitativamente a la capacidad de la TVLR para generar públicos masivos no segmentados mientras, por el contrario, la CATV mostrará creciente fortaleza en los sectores de ingresos medios y altos de los centros urbanos y en su capacidad de generar simultáneos y múltiples públicos segmentados.

- En materia programática, la TVLR deberá intentar aprovechar sus ventajas comparativas propias, e la programación nacional en general y programación periodística en particular, abandonando

$\mathrm{Ni}$ los cambios tecnológicos, ni la expansión de la televisión por cable, ni siquiera el desarrollo de la súper carretera electrónica y la televisión interactiva, harán desaparecer a la televisión de libre recepción, la que es posible que crezca en términos absolutos, aunque pierda posiciones relativas. progresivamente las áreas donde las ventajas comparativas mayores las tienen la CATV, como es el caso del cine, las series y la programación especializada para segmentos específicos (infantil, deportiva, etc.).

- En materia de publicidad comercial, la TVLR tendrá que aprovechar su ventaja competitiva para avisadores de bienes o servicios de consumo masivo, de decisión de compra no segmentada y de precios absolutos medio-bajos y bajos, en respuesta al creciente interés de los avisadores de bienes y servicios de las características precisamente opuestas por la CATV.

- Es razonable esperar que el número de canales de TVLR de alcance nacional y de programación general no segmentada será de dos o tres a los más, siendo previsible que ellos sean TV-UC, TVN y, eventualmente, Megavisión.

- Lo anterior llevará a los restantes canales de TVLR a reorientarse hacia una programación segmentada y costos de explotación menores, sea esta segmentación de carácter regional (UCV-TV, Telenorte y los Centros Regionales de TVN), de grupos hetarios específicos (futuro Canal 2) o de otra naturaleza aún no bien definida (Chilevisión y La Red). 
A lo largo de los últimos años, ha habido un constante aumento del número de señales satelitales internacionales disponibles para la CAIV y contratadas por la industria. Es previsible que este aumento continúe, al menos hasta el límite financiable por el precio de los servicios básicos de la CATV.

- Por último, es posible prever una tendencia creciente a la concentración en pocas manos de propiedad de MCS de distinto tipo ("Multimedia"), con una fuerte presencia de empresas transaccionales extranjeras.

\section{PERSPECTIVA DE DESARROLLO DE LA TELEVISION POR CABLE}

Me corresponde ahora, en esta tercera parte de a exposición, referirme a la perspectiva de desarrollo de la CATV en Chile, lo que haré tocando fundamentalmente cuatro puntos: el desarrollo cuantitativo, el desarrollo programático, el desarrollo tecnológico, los procesos de globalización y de convergencia, para extraer al final algunas conclusiones.

\section{El desarrollo cuantitativo de la CAIV}

Antes de examinar las cifras de la CATV chilena, conviene ponerlas en perspectiva, analizando sumariamente el desarrollo internacional de esta industria, tanto en los paises desarrollados como en América Latina.
- Existen notables diferencias entre la penetración de la CATV entre paises de la misma región, siendo casos extremos Bélgica (58\%) y Corea (menos de $1 \%$ ) en Otros.

\section{Desarrollo de la CATV en los países latinoamericanos}

En el Cuadro $\mathrm{N}^{\circ} 2$, aparecen las mis-

\section{Desarrollo de la CAIV en los países desarrollados}

El Cuadro $\mathrm{N}^{\circ} 1$ contiene las cifras de hogares con TVLR (HG TVLR), de hogares cableados o "home-passed" (HG H.P.), y de hogares con CATV (HG CATV), así como los porcentajes de penetración de la CATV, tanto de HG H.P. sobre HG TVLR, como de HG CATV sobre HG H.P. y HG TVLR, para una muestra representativa de paises desarrollados.

De las cifras del Cuadro $\mathrm{N}^{\circ} 1$ (al final de este artículo), pueden obtenerse las siguientes conclusiones sobre la CATV en esta muestra de países desarrollados:

- La penetración total de la CATV es de un $37 \%$ promedio en relación a la TVLR, producto de una tasa de cableado de un $57 \%$ y de una tasa de penetración de un $65 \%$.

- La mayor penetración de la CATV se da en Norteamérica con un $63 \%$, seguida de Europa Occidental (34\%), Europa Oriental $(15 \%)$ y Otros $(10 \%)$.
De las cifras del Cuadro $\mathrm{N}^{\circ} 2$, pueden obtenerse las siguientes conclusiones sobre la CATV en los países latinoamericanos:

- La penetración total de la CATV es de un $11 \%$ promedio en relación a la TVLR, producto de una tasa de cableado de un $19 \%$ promedio y de una tasa de penetración de un $54 \%$.

- Estas cifras son sensiblemente inferiores a las de la muestra de paises desarrollados, tanto en la penetración total ( $37 \%$ contra $11 \%$ ), producto sobre todo de la diferencia en la tasa de cableado $(57 \%$ contra $19 \%$ ) y, algo menos, de la diferencia en la tasa de penetración $(65 \%$ contra $54 \%$ ).

- La mayor penetración de la CATV se da en México (63\%), seguida de Sudamérica (105) y América Central (también $10 \%$ ).

- Existen notables diferencias entre la penetración de la CATV entre paises de la misma región, siendo casos extremos Argentina (53\%) y Brasil (1\%) en 
Sudamérica, y República Dominicana (24\%) y Nicaragua (3\%) en América Central.

- Chile ocupa una posición levemente inferior al promedio de Latinoamérica en penetración total $(95 \%$ contra $11 \%$ ), ya que aunque tiene una mayor tasa de cableado ( $23 \%$ contra $19 \%$ ), tiene una menor tasa de penetración $(40 \%$ contra $54 \%$ ).

- Para apreciar el potencial de desarrollo futuro de la CATV en Chile bastaría con señalar que, para llegar a tener los mismos indicadores que Argentina, necesitaría más que triplicar el número de hogares cableados, de 800.000 a 2.520.000, y casi multiplicar por seis el número total de hogares abonados, de 320.000 a 1.890 .000 .

\section{Desarrollo de la CAIV en Chile}

A la luz de lo anterior, para examinar el desarrollo actual y, sobre todo, las perspectivas futuras de la CATV en Chile debemos referirnos a dos temas: el número de actores principales en la industria, por una parte, y el número de abonados y la tasa de su crecimiento para cada uno de ellos. El número de actores en la CAIV
chilena
En los dos últimos años se ha producido una fuerte disminución del número de actores en la CATV chilena, debido al proceso masivo de compra de medianos y pequeños operadores locales por redes nacionales, con lo cual la situación puede hoy día resumirse de la siguiente manera:

- En el Gran Santiago operan:

-Tres empresas de CATV que usan línea física para la distribución de sus señales a los abonados, que son (por orden alfabético) Cablexpress, Intercom y Metrópoli, y

-Una empresa de servicios limitados de televisión, que utiliza microondas codificadas para la distribución a sus abonados (TV-Max, de VTR), que ha iniciado recientemente construcción de líneas físicas.

- En Regiones operan:

-Dos importantes cadenas de CATV, de la misma propiedad de empresas que operan en Santiago (VTR y Metrópolis);
-Dos cadenas de CATV de carácter regional, STX en las Regiones Primera, Segunda y Tercera, y UIH en las Regiones Cuarta y Quinta, siendo al parecer un hecho que la segunda ha adquirido ya el control de la primera, $y$

-Una docena de operadores de CATV en ciudades medianas y pequeñas.

\section{Tamaño relativo y tasa de crecimiento de los actores de CAIV}

Por razones que quizás alguna vez fueron justificadas, es extremadamente difícil tener cifras confiables del número de abonados de cada CATV en el pasado. Aún hoy, en que el pretender mantener reserva de lo que es un "secreto a voces" me parece carente de toda justificación, es aventurado dar cifras. Las tasas de crecimiento del número de abonados de las empresas de CATV (a lo menos en Santiago), son, al parecer, más confiables que las cifras absolutas. De ese crecimiento es posible obtener las siguientes conclusiones:

- El mercado de la CATV en Santiago está creciendo a una tasa muy acele-
La búsqueda de la diferenciación y de las eventuales ventajas competitivas derivadas de ella, lograda a través de la producción de señales locales exclusivas de cada CAIV, se ha transformado así en una de las variables estratégicas en el desarrollo futuro de la industria de la CATV. 
El presente y el futuro de la CATV, en el mundo y también en Chile, no sólo nos ha traído y traerá cambios en el número y peso relativo de las empresas y en sus contenidos programáticos sino, también, nuevos e importantes desarrollos tecnológicos, algunos de los cuales ya se están materializando, mientras otros se ven cada vez más cercanos.

rada, y hay buenas razones para suponer que en Regiones la situación es similar.

- El crecimiento del mercado total de la industria, unido al hecho de que, por poco tiempo más, hay más áreas en las que opera una sola empresa que áreas en que se sobrepongan dos o más, ha permitido que todas las empresas crezcan, al menos en Santiago, fundamentalmente sobre la base de nuevos abonados y no sobre la base de traspaso de abonados de una empresa a otra.

- Sin perjuicio de ello, hay notables diferencias en las tasas de crecimiento de las distintas empresas, $\mathrm{y}$

- Lo anterior, unido a que durante lo que resta del año se sobrepondrá el cableado de dos o tres empresas en casi todas las áreas del Gran Santiago, hace previsible que se observarán cambios importantes en el tamaño relativo de las empresas que operan en Santiago, siendo más que probable que la línea de crecimiento tienda a disminuir sus pendientes actuales y, eventualmente y para alguna empresa, a aplanarse.

\section{El desarrollo programático de la CATV}

A esta fecha, los servicios básicos de la CATV chilena, que son los únicos que presta hoy en día, se componen de una treintena de señales satelitales internacionales, unas pocas señales locales propias de cada CATV y seis señales correspondientes a los canales de la TVLR.

Dado que la industria de la CATV, en todo el mundo y también en Chile, descansa sobre una política invariable de que las señales satelitales internacionales no son concedidas en exclusividad a ningún operador de CATV, el componente internacional de la programación de la CATV chilena se caracteriza por dos hechos: el aumento de señales y la esencial homogeneidad de la programación internacional de todas las empresas de CATV, al menos las mayores.

A lo largo de los últimos años, ha habido un constante aumento del número de señales satelitales internacionales disponibles para la CATV, todas las más im-

\section{Las señales satelitales internacio- nales}

portantes de las cuales han sido contratadas por la industria. Es previsible que este aumento continúe, al menos hasta el límite financiable por el precio de los servicios básicos de la CATV.

Lo anterior ha llevado a que en Chile, como en todos los paises en que los operadores de CATV ofrecen sólo un servicio básico, exista una casi perfecta homogeneidad en la oferta internacional de los distintos operadores que, con muy escasas diferencias, ofrecen todos las mismas señales satelitales.

La esencial semejanza entre todos los operadores de CATV en materia de señales satelitales, y el hecho de que estas han constituido tradicionalmente el mejor atractivo de la industria a sus abonados, ha traído aparejada dos consecuencias importantes:

- Por una parte a que, desde el punto de vista del abonado, actual o potencial, los distintos operadores de CATV sean lo que se conoce, en términos de teoría económica, como "bienes o servicios (casi) perfectamente sustituibles", ya que -a igualdad de precios y calidad de servicio- le es casi indiferente al cliente a cual CATV se abona; correlativamente, la "lealtad de marca" es casi inexistente.

- Por otra parte, lo anterior ha obligado a todos los operadores de CATV a buscar ansiosamente el elemento diferenciador, que le den a su servicio una ventaja sobre los competidores. Dada las características de las señales satelitales, ello sólo puede ser logrado por la vía de las señales locales y de propiedad y derechos exclusivos de una CATV en particular. 


\section{Las señales locales exclusivas}

La búsqueda de la diferenciación y de las eventuales ventajas competitivas derivadas de ella, lograda a través de la producción de señales locales exclusivas de cada CATV, se ha transformado así en una de las variables estratégicas en el desarrollo futuro de la industria de la CATV.

Hasta el momento al menos, el tipo de señales nacionales exclusivas que se han creado, además de los "canales de servicio" de las CATV, han sido básicamente señales dedicadas a programas de conversación (talk shows), de bajo costo y de escasa, aunque a veces selecta, audiencia.

Este panorama comienza a cambiar, con recientes experiencias de señales de cine armadas localmente y otras iniciativas, que parecen apuntar más certeramente al objetivo estratégico de una diferenciación ante la audiencia. Con todo, la experiencia más audaz en su concepción, así como la mayor apuesta hecha en el mercado chileno, fue la compra por parte de Cablexpress a la ANFP de derechos exclusivos por 168 partidos de los Campeonatos Oficiales 1995-1997, en todos los cuales juegan los tres clubes más populares. Es por ello que, pese a estar este tema vinculado al grupo de Empresas que dirijo, deseo compartir con ustedes algunos antecedentes sobre el efectivo valor estratégico de un canal nacional exclusiva de atracción masiva, después de referirme a las limitantes que tiene, al menos por el momento, este.

\section{Limitantes a la producción de señales nacionales de CATV}

Con toda la importancia estratégica que la industria de CATV da a la señales nacionales como elementos de diferenciación, avaladas por la experiencia concreta que compartiré con ustedes más abajo, es necesario, sin embargo, señalar que hay fuertes limitantes a la producción de este tipo de señales.

La primera limitante la constituye la reducida capacidad de producción propia de las CATV, en términos de instalaciones y recursos técnicos y humanos. Pero la limitante más importante la constituye el alto costo, total y por abonado, de la producción nacional, especialmente si progresamos más allá de la etapa elemental de los meros programas "de conversación". En efecto, las CATV chilena cobran hoy día a sus abonados una tarifa por un sólo servicio básico, igual para todos los abonados, lo que limita seriamente la posibilidad de emprender producciones de más alto vuelo. Es por ello que creo que es una hipótesis razonable que la CATV chilena, más temprano que tarde, abandonará este esquema único, para entrar a financiarse mediante el desarrollo de servicios diferenciados, con precios diferentes, destinados a sectores específicos de sus abonados. A esa materia me referiré más

La cobertura potencial de la televisión de libre recepción será de dos a tres veces mayor que la de televisión por cable. abajo, después de examinar un caso concreto de experiencia de "diferenciación de marca" en el contexto actual de existencia sólo de servicios básicos.

\section{Diferenciación de marca y fútbol profesional}

La oferta que en su momento hizo Cablexpress a la ANFP, US\$ 16.500 en tres años, fue una auténtica sorpresa para todos, así como "una locura" para muchos. Debo confesar que a mí -que en esa época trabajaba en TVN- la oferta de Cablexpress también me sorprendió. Por eso es importante examinar ahora los resultados de esta estrategia.

\section{Efectos en el crecimiento de la empresa}

Una forma de medir los resultados de esta estrategia es a través de su efecto en el crecimiento del número de abonados; desde este punto de vista los resultados son francamente espectaculares: en efecto, la tasa promedio de crecimiento del número de abonados tiene un claro "punto de quiebre" en diciembre de 1994, en que dicho promedio crece en un $80 \%$ en relación al de los meses anteriores. 


\section{Efectos sobre la audiencia}

Otra forma de medir los resultados de esta estrategia es a través de su efecto en la audiencia de la empresa y de sus diferentes señales. A este respecto, dispongo de los datos provenientes de una sucesión de seis encuestas telefónicas coincidentales, hechas a los abonados de Cablexpress por la empresa de estudios de mercado "Red Cable Telemarketing". Las encuestas se realizaron en tres días sábados y tres días domingos, a las horas de transmisión habitual del fútbol. Se abarcó un total efectivo de 3.480 receptores de televisión en 1.736 hogares. Las encuestas se hicieron en días que permitieran comparar las audiencias en días sin transmisión de fútbol, con transmisión de partidos "normales" y con transmisión de partidos "clásicos" (por ejemplo, Colo Colo vs. Universidad de Chile).

En el Cuadro $\mathrm{N}^{\circ} 3$ se consigna el "rating" (porcentaje sobre el total de televisores, estén encendidos o no), de cuatro grupos de señales de Cablexpress: señales de TVLR; señal de fútbol ("Superdoce"): señales satelitales de cine, y otras señales satelitales. El cuadro diferencia entre los "rating" de días sin fútbol, días con partidos "normales" y días con partidos "clásicos"; además consigna las diferencias que, parada cada grupo de señales, se produce como consecuencia de esas distintas programaciones de fútbol.

\section{Conclusiones sobre el efecto en el rating}

- El "rating" total de Cablexpress los días sábado y domingo en la tarde es superior a un $50 \%$, lo que excede el "rating" en esos mismos días y horas de cualquier canal de TVLR y de todos ellos en conjunto.

- El "rating" total no se ve afectado por la transmisión de fútbol, ni siquiera de partidos "clásicos" aunque sí, se altera fuertemente el "rating" de las distintas señales.

- El "rating" del fútbol es de un $25 \%$ en partidos "normales" y de un $40 \%$ en partidos "clásicos", viéndose afectado en esos casos el "rating" del conjunto de señales de cine) bajas de 18 y $21 \%$, respectivamente), de las restantes señales satelitales (bajas de 6 y $10 \%$, respectivamente) y de las señales de los canales de TLVR (bajas de 5 y $10 \%$, respectivamente).

- En días sin fútbol, el mayor "rating" lo tienen las señales de cine (25\%), seguidas de los canales de TLVR (15\%) y las restantes.

\section{Resultados de share}

El Cuadro $\mathrm{N}^{\circ} 4$ entrega los resultados de "share" (porcentaje sobre el total de televisores encendidos), para los mismos grupos de señales y tipos de días que el Cuadro $\mathrm{N}^{\circ} 3$.

\section{Conclusiones sobre el efecto en el share}

- El "share" de las distintas señales se altera fuertemente por la transmisión de fútbol, en especial de partidos "clásicos".

- El "share" del fútbol es de un $50 \%$ en partidos "normales" y de un $75 \%$ en partidos clásicos", viéndose afectado en esos casos el "share" del conjunto de señales de cine (bajas de 32 y $39 \%$, respectivamente), de las restantes señales satelitales (bajas de 10 y $19 \%$ respectivamente) y de las señales de los canales de TVLR (bajas de 8 y $18 \%$, respectivamente).

- En días sin fútbol, el mayor "share" lo tienen las señales de cine (46\%), seguidas de los canales de TVLR (27\%) y las restantes señales satelitales (26\%).

\section{Evaluación de la experiencia}

Aunque, por cierto, no ha transcurrido el tiempo suficiente para evaluar completamente la experiencia de "diferenciación de marca" de Cablexpress a través de su señal local exclusiva "Superdoce", pueden sacarse desde ya algunas conclusiones:

- El efecto de "diferenciación de marca" producido a Cablexpress por su señal "superdoce" ha sido plenamente logrado, en niveles no comparables con los de las señales locales de otras CATV.

- La transmisión de partidos de fútbol en general y, más marcadamente aún, de partidos "clásicos" altera signifi- 
troncales, conectando los "Head End" de cada empresa con sus modos de distribución y/o "Head End Secundarios", permitiendo la transmisión de una cantidad mucho mayor de señales, a distancias más largas y con menores pérdidas de calidad.

Pronto también la CATV chilena se verá beneficiada con la disponibilidad de fibra óptica interurbana, sea de su propiedad o arrendadas a empresas de telecomunicaciones, permitiendo ampliar la oferta de señales y servicios en ciudades medianas y pequeñas y haciendo innecesaria la construcción de "Head Ends" en cada una de ellas.

\section{Ampliación del ancho de banda de transmisión}

Paralelamente a la introducción creciente de la fibra óptica en sus plantas externas, las CATV han ampliado sus anchos de bandas de transmisión, de 450 ó 500 $\mathrm{MHz}$ a $750 \mathrm{MHz}$ o más. Con ello se amplía aún más el número de señales que pueden recibir los abonados y, además, se adquiere la capacidad para prestar otro tipo de servicios, tanto en televisión como en telefonía, transmisión de datos y otros.

\section{Bidireccionalidad de las redes}

La fibra óptica, las bandas anchas de transmisión y el reemplazo de algunos elementos electrónicos u opto-electrónicos, activos o pasivos, permitirá la bidireccionalidad de las redes de la CATV, esto es ellas están habilitadas no sólo para trans- mitir señales desde la empresa a los hogares de los abonados, sino también desde los hogares a su CATV, lo que abrirá camino a la interactividad, de la televisión y de los otros servicios que ofrezca la CATV.

\section{Interactividad}

Aparece como el "gran futuro" de la CATV, apoyada en las llamadas "super carreteras electrónicas”, redes de fibra óptica, banda ancha y bidireccionales, capaces de prestar servicios interactivos de televisión, de telefonía, de transmisión de datos, de "telemarketing" y muchos otros.

\section{Los procesos de globalización y de convergencia}

Dos grandes fuerzas, dominan el futuro de las telecomunicaciones y de la televisión, sea TVLR o CATV: la globalización y la convergencia.

\section{Globalización de las empresas}

En el proceso por el cual las empresas de todos los paises -empujadas por la competencia y los altos costos de las nuevas tecnologías- se van integrando progresivamente en conglomerados multinacionales, que operan en muchos mercados, aprovechando economías de escala y transfiriendo su "know how" a sus empresas filiales o asociadas.

Este proceso están en pleno desarrollo en Chile, con una fuerte presencia de capital extranjero en las industrias, entre otras, de la computación, las telecomunicaciones y los MCS en general, en especial la televisión. En el caso chileno, a los factores mundiales que operan se agregará, para acelerar este proceso, la existencia de uno de los regímenes de inversión extranjero más liberales del mundo, en especial en materia de MCS. En esta última materia, Chile es casi la excepción mundial, en el sentido de no poner limitaciones a la existencia y proporción de propiedad extranjera en su MCS, pese a no haber reciprocidad en relación a la inversión de chilenos en los MCS de otros paises.

\section{Convergencia de las industrias}

Es el proceso por el cual las diferencias industrias -empujadas por la necesidad de competir y las nuevas tecnologías, en especial la digitalización- van convergiendo progresivamente en conglomerados de empresas o empresas integradas, que operan mercados de muchos productos y/o servicios diferentes.

Este proceso de convergencia está llevando a que desaparezcan progresivamente las barreras que antes separaban a las industrias de la computación, de las telecomunicaciones en sus múltiples variedades, y de los MCS, en especial la televisión.

\section{Conclusiones sobre la perspectiva de desarrollo de la CATV}


cativamente la audiencia de las señales, tanto en términos de "rating" como de "share".

- Si se piensa en los precios a que se efectuaron las transacciones de los paquetes accionarios mayoritarios de dos importantes CATV del mercado hechas el año pasado (superiores a los US\$ 1.700 por abonado), la inversión de Cablexpress de US\$16.500.000 se vería “cubierta”, en términos de aumento del valor económico de la empresa, sólo por el número de nuevos abonados conseguidos en apenas dos meses.

\section{El desarrollo de nuevos productos $y$ servicios}

La necesidad de competir y diferenciarse, así como los altos costos de la producción nacional de calidad, llevarán a las CATV chilena, en mi opinión, a ofrecer nuevos productos y/o servicios.

Todos ellos suponen como prerrequisito la codificación de todas o parte de las señales de una CATV, con la consiguiente inversión en codificadores en sus "Head End" y, sobre todo, de decodificadores en los hogares de sus abonados.

Se trata de inversiones considerables, pero que pueden ser rentables a mediano plazo, al permitir el desarrollo de nuevos productos y servicios que, satisfaciendo necesidades específicas de los abonados, signifique nuevos e importantes ingresos para las CATV.

\section{Señales de pago mensual adicio- nal (canales premium)}

Este servicio consiste en la oferta al abonado de uno o más canales no incluidos en el servicio básico, a cambio de un pago mensual adicional. Esta modalidad, existente en la mayoría de los paises, se conoce como canales "premium" y permite ofrecer a otro nivel de programación, extranjera y/o nacional, que no podría ser financiada mediante el pago del servicio básico, sin encarecerlo mucho, afectando así el número de abonados, en especial de los de ingresos medios o mediosbajos.

\section{Programas específicos de pago único adicional}

Este servicio consiste en la oferta al abonado de uno o más canales no incluidos en el servicio básico, a cambio de un pago mensual adicional. Esta modalidad, existente en la mayoría de los paises, se conoce como canales "premium" y permite ofrecer otro nivel de programación, extranjera y/o nacional, que no podría ser financiada mediante el pago del servicio básico, sin encarecerlo mucho, afectando así el número de abonados, en especial de los de ingresos medios o mediosbajos.

\section{Programas específicos de pago único adicional}

Este servicio consiste en la oferta al abonado de uno o más programas específicos, no incluidos ni en el servicio básico ni en los canales "premium", a cam- bio de un pago único adicional por cada programa. Este servicio, existente ya en muchos paises, se puede ofrecer en dos formas diferentes, a saber:

- Sobre la base de una oferta de un programa determinado que hace el CATV a todos sus abonados, para ser transmitido en un día y hora determinado, modalidad que se conoce como "pay per view", o bien

- Sobre la base de una oferta de una "librería de programas" que el CATV pone a disposición de sus abonados, quienes escogen no sólo el programa sino el día y hora en que cada uno de ellos quiere verlo, modalidad que se conoce como "pay on demand".

\section{El desarrollo tecnológico de la CATV}

El presente y el futuro de la CATV, en el mundo y también en Chile, no sólo nos ha traído y traerá cambios en el número y peso relativo de las empresas y en sus contenidos programáticos sino, también, nuevos e importantes desarrollos tecnológicos, algunos de los cuales ya se están materializando, mientras otros se ven cada vez más cercanos.

\section{Incorporación de la fibra óptica}

Se trata de un cambio que se ha iniciado ya, desde fines del año pasado, en las plantas externas de las principales CATV en Santiago, en que la fibra óptica está reemplazando al cable coaxial, en especial en las líneas troncales y sub- 
En apretada síntesis, mis conclusiones sobre el desarrollo futuro de la industria de la CATV en Chile podrían resumirse en los siguientes puntos:

1. La industria de la CATV en Chile se encuentra en una primera etapa de su desarrollo, teniendo tasas de penetración efectiva muy inferiores a las de paises desarrollados y, también, inferiores a los de otros paises latinoamericanos, singularmente Argentina.

2. La industria del CATV seguirá creciendo este año, tanto en Santiago como en Regiones, a tasas muy elevadas; ello permitirá por un tiempo al menos, que todas las empresas crezcan, especialmente sobre la base de incorporar abonados nuevos; sin embargo, el próximo año la situación evolucionará y, si bien la industria seguirá creciendo, aunque a tasas más moderadas, el crecimiento, estancamiento o disminución de cada empresa dependerán de su capacidad de ofrecer un producto diferenciado.

3. Este elemento de diferenciación no serán las señales satelitales, esencialmente las mismas para todas las empresas, sino las señales locales exclusivas, en especial las que puedan verdaderamente marcar una diferencia. Todas las empresas CATV están intentando desarrollar estos elementos diferenciadores y ya hay, a lo menos, una experiencia claramente exitosa al respecto. Sin embargo el desarrollo de señales locales exclusivas estará limitado por los altos costos de producción, necesarios para productos de calidad, los que no serán diferenciables con el cobro de un único y común servicio básico a todos los abonados.

4. Por ello, la CATV chilena deberá intentar desarrollar productos diferenciados, de pago adicional, para satisfacer necesidades especiales de grupos de abonados específicos. Entre otros productos estarán los "canales premium", el "pay per view", el "pay on demand" y/u otros.

5 . Se están incorporando ya importantes avances tecnológicos, como la fibra óptica y la banda ancha de transmisión, y se avecinan la bidireccionalidad de las redes y la televisión interactiva, todo ello dentro del marco de un doble proceso -mundial y chileno- de globalización y convergencia, que llevarán a las "super carreteras electrónicas", que transportarán los servicios de industrias antes separadas y que serán administradas crecientemente por conglomerados multinacionales.

\section{CONCLUSIONES FINALES}

Mis conclusiones finales sobre la perspectiva de desarrollo de las industrias de TVLR y de CATV son muy simples:

1. Existe un gran futuro, tanto para la TLVR comino para la CATV, en un mundo que, no por ancho, nos será necesariamente ajeno.

2. Ni la TVLR desaparecerá, ni necesariamente decrecerá en términos absolutos, aunque perderá terreno relativo frente a la CATV.

3. La CATV recién inicia su desarrolla y en su futuro se avistan oportunida- des excepcionales de desarrollo cuantitativo, programático, técnico y económico.

4. Por lo tanto, todos tenemos mucho trabajo por delante. Se necesitará visión de futuro, talento... y también dinero.

* Ponencia presentada en la conferencia "Televisión Satelital y por Cable en Latinoamérica, 1995”, realizada en Santiago los días 5, 6 y 7 de junio de 1995). 